Published in final edited form as:

J Law Med Ethics. 2014 December ; 42(Suppl 2): 17-25. doi:10.1111/jlme.12183.

\title{
Envisioning a Transdisciplinary University
}

\author{
Leigh Carroll*, \\ Institute of Medicine, Washington, DC, USA
}

Mohammed K. Ali, MBChB, MSc, MBA,

Hubert Department of Global Health, Emory University, Atlanta, GA, USA

Patricia Cuff ${ }^{\star}$, Institute of Medicine, Washington, DC, USA

Mark D. Huffman, MD, MPH,

Assistant Professor of Preventive Medicine and Medicine-Cardiology, Northwestern University Feinberg School of Medicine, Chicago, IL, USA

Bridget Kelly, MD, PhD*, Institute of Medicine, Washington, DC, USA

\section{Sandeep P. Kishore, MD, PhD,} Young Professionals Chronic Disease Network, YP-CDN, Boston, MA, Yale University, New Haven, CT

\section{K.M. Venkat Narayan, MD, MSc, MBA, Hubert Department of Global Health, Emory University, Atlanta, GA}

Karen R Siegel, MPH, and

Hubert Department of Global Health, Rollins School of Public Health, Emory University Nutrition and Health Sciences, Laney Graduate School, Emory University, Atlanta, GA

\section{Rajesh Vedanthan, MD, MPH}

Zena and Michael A. Wiener Cardiovascular Institute, Mount Sinai Medical Center, New York, NY

\section{Abstract}

Achieving social and economic growth requires collaboration, especially in global health. If universities are to improve health globally, they will need to train students and to support faculty who can effectively collaborate with those from other disciplines and cultures.

\section{Introduction}

Thomas Jefferson once suggested that a university should be designed so that "the whole arranged around an open square of grass and trees would make it, what it should be in fact, an academical village." 1 Unfortunately, the current academic setting often bears little resemblance to a village that pools its residents' strengths to learn and solve problems.

\footnotetext{
Correspondence to: Bridget Kelly, Institute of Medicine, 500 Fifth St, NW, Washington, DC 20001, (202) 334-2533, bkelly@ nas.edu. The authors are responsible for the content of this article, which does not necessarily represent the views of the Institute of Medicine
} 
Instead, universities often seem to take pride in their collections of segmented and highlyspecialized disciplines functioning in their own silos - a structure that often serves as an impediment to effective collaboration. Is it possible to change this and design universities that are arranged around an open square of ideas - allowing human creativity, compassion, and critical thinking to form a community that can address society's great and pressing problems?

In August 2012, the Institute of Medicine and the interdisciplinary Young Professionals Chronic Disease Network hosted a meeting on Transforming Global Health Education in the $21^{\text {st }}$ Century at Emory University. The focus was global health, as it is an interest of the organizers and one of the many areas in which work across disciplines is especially vital. For example, tackling tobacco control in New York City required the voices and cooperation of many groups and individuals, including clinics, media, politicians, epidemiologists, sociologists, human rights advocates, businessmen, and community organizers. In Bangladesh, reducing harmful smoke from indoor cook stoves in homes that rely primarily on firewood for cooking, and thus improving the health of their inhabitants, depended on the cooperative efforts of community members, engineers, educators, health professionals, environmentalists, women's empowerment groups, and anthropologists. And most universally, raising a child does requires the efforts of family members, teachers, doctors, neighbors, coaches, and others.

The 2012 meeting brought together a diverse group of 30 participants $^{2}$ (students, faculty, and professionals) representing a variety of institutions and disciplines (medicine, public health, urban design, theology, humanitarian studies, fine art, biological sciences, graphic design, law, economics). The goals of the meeting were to (1) brainstorm ways in which universities can become environments where collaborative work across disciplines thrives and (2) compile different approaches and tools for engagement between disciplines at universities. Participants focused specifically on how universities can be an incubator in which many disciplines work together to improve the health of communities.

This paper reflects many of the ideas shared at this meeting, as well as thoughts that have arisen among participants after the meeting. The findings shared in this paper are relevant to the goals of the University of Maryland, Baltimore (UMB) 2013 roundtable and are set forth to help move the UMB project forward.

Although the discussion of the 2012 meeting centered on working across disciplines, participants did not explicitly differentiate between multi-, inter-, and transdisciplinary collaboration. Instead, participants saw these as related concepts that fall somewhere on a continuous spectrum. This paper uses "transdisciplinary" as a broad term to encompass the entire spectrum of work across disciplines, and recognizes that others have done more extensive work to describe and differentiate the concept. ${ }^{3}$

This paper is a discussion of what it might take to create transdisciplinary university environments that provide a safe space for risk-taking, facilitate meetings between people and ideas, and instill a sense of the limits of individual or isolated knowledge and perspectives. In other words, this environment would acknowledge independence within an 
interdependent framework. After a brief discussion of the current university model, this paper focuses on two questions: First, what are key characteristics of a transdisciplinary university? Second, what can people do to expand these university environments?

\section{The Current Environment}

Universities, in charge of training tomorrow's cadre of leaders to tackle critical global issues, are often not ideally structured to approach problems from many angles and perspectives. Existing educational and organizational structures tend to "funnel" students down one track or another, with the ultimate goal of creating specialized professionals able to prosper within their specific fields. This poses two problems: first, "specialists" may go through and complete training with limited exposure to the larger context of problems or the perspectives of different types of specialists working on similar issues. This leads to a focus on solving the immediate issues while limiting the ability to solve the underlying structural and contextual framework creating the problem. Second, by focusing on specialists, universities are less likely to nurture multidisciplinary teams or individuals who focus on working across disciplines. People who work and think across disciplines may not be encouraged or rewarded for their work and are often regarded as unfocused "dabblers", yet their transdisciplinary knowledge and experience has significant creative value.

Many universities and academic programs are already taking bold steps toward becoming more transdisciplinary (see some examples in Figure 1) and many of the ideas expressed in this paper are university characteristics that faculty and students have already begun to adopt.

\section{What Are The Characteristics Of A Transdisciplinary University?}

Our vision is that universities become transdisciplinary environments-places that provide a safe space for risk-taking, facilitate collisions between people and ideas, and instill a sense of the limits of individual or isolated knowledge and perspectives. These universities would not necessarily do away with disciplines, but would create environments that allow for a variety of approaches to problem-solving, and for the healthy and respectful intermingling of disciplines.

In the following several sections, the paper focuses on specific areas of change that universities must address to do this. In particular, universities will need to embrace and foster a new mentality of problem-solving ("Mentality"), expand the scope of its training to include and support many types of learners and doers ("People"), and increase the frequency with which they interact with each other across disciplines ("Groups").

\section{Mentality}

A transdisciplinary university community allows its members to adopt a more flexible mentality —one that permits people to step outside of the bounds of typical thinking and accept that there are limits to what is known in individual fields or from individual perspectives. In short, transdisciplinarity requires more humility that recognizes the boundaries of what single disciplines can achieve and more openness to other perspectives. 
Humility-To function well, a transdisciplinary university community will require and foster humility. There are several angles to this humility, including an awareness of ignorance and the active avoidance of the delusion of knowing it all and an openness to change.

First, champions of the transdisciplinary environment will be aware of their ignorance and the limits to individual and group knowledge. As Bertrand Russell said, "the whole problem with the world is that fools and fanatics are always so certain of themselves, and wiser people so full of doubts." ${ }^{4}$ Humans will never fully understand the world and each other, nor know what we do not know unless we allow ourselves and our thinking to be challenged.

Second, people in a transdisciplinary environment will be open to change. As Einstein is thought to have said, "we will never solve problems by using the same level of consciousness we used when we created them." ${ }^{5}$ What people know and have invested in is not necessarily the best way to solve problems. Professional hierarchies will change, power will shift, and territory will be redistributed. If people truly care about the problems they are trying to solve, they must adapt to new ways of doing things.

Openness-Many disciplines have become more constrictive, rather than open. For example, individuals may be reluctant to bring unscientific, personal, experiential, and emotional perspectives into academic discussions. It is assumed that, although varied interests relate naturally to the private life of a person, they do not rightfully relate in polite academic society.

Some of this may stem from different understandings of what constitutes "evidence" or sound methodology across disciplines. For example, many people experience some sort of "truth" when immersed in music, and this may be different than the type of "truth" discussed in a philosophy class, and different yet again for a math class.

Many different types of evidence and methodologies contribute to understanding, and the problem arises when disciplines put their knowledge at the top of a hierarchy. If people are shy to share insight from one discipline that they think may be disregarded in another discipline, many unexpected connections may be missed. For example, developing a physical model of hyperbolic space had eluded humans for ages until mathematician Daina Taimina used her experience with handicrafts to develop a model through crochet. ${ }^{6}$

Less rigid and restrictive discipline is not only needed within individuals, but also within groups and collaborations. Like-minded individuals collaborate because it is easy and frequently productive, and perhaps more intuitive than reaching beyond those who share a similar perspective. Stepping out of what is "proper" in one's field can be uncomfortable but perhaps collaborators should embrace learning from the knowledge and perspectives of unusual "outsiders". The development of relationships is a key component of successful growth, both within a discipline but particularly across disciplines.

In a truly transdisciplinary university environment, students and faculty would not feel the pressure to limit themselves to the boundaries of their disciplines; rather, there would be a 
certain freedom to explore their hunches and seek out people who might not fit their typical teammate mold.

People

A transdisciplinary environment should support many types of learners and doers, and universities may need to consider ways of identifying these different types of people and matching them with people and projects that would benefit from their insight. To generalize very broadly, a university should support the following types of people:

- $\quad$ People who specialize and are completely immersed in one discipline. These people do not necessarily prioritize linking disciplines or understanding how their work fits into a broader context, but are dedicated to delving into complexities within their discipline. They should be supported by university structures that allow them to be easily identified and reached when their specialized knowledge is needed for something broader.

- $\quad$ People who specialize in a discipline but are aware of context. These people have deep knowledge in one discipline but want to understand to some extent how their work fits into a broader context. These people need to be supported with opportunities that expose them to the context of the problems that need their expertise. For example, the engineer of a cook stove that is used to reduce smoke in homes that previously used firewood for cooking would need to understand that his/her product will only be adopted by communities if it is culturally and economically appropriate in that context.

- $\quad$ People who specialize in two or three disciplines and bridge them together. These people need to be supported by programs that are flexible enough to allow for specialization in multiple disciplines. For example, a person interested in the intersections of spirituality or religion and public health could do a joint masters program in public health and theology.

- $\quad$ People who specialize in context thinking and connecting. These are people who do not specialize in a current discipline but see things in less traditional categories. They are particularly skilled in recognizing how things relate to each other and in bringing together seemingly disparate bodies of knowledge. These people often are seen as "dabblers". In reality, though, they may have just as much depth of knowledge as a specialist, but from a perspective that is not formally recognized. In fact, these individuals may be exploring the disciplines of the future. These "connectors" are the people who face the most difficulty in some fields and universities (though they are frequently valued more in liberal arts schools). They are often viewed as lacking focus, especially when they take this approach early in their careers rather than first specializing narrowly. These people need universities that value their transdisciplinary knowledge and experience and that allow them to put their knowledge to use through projects that do not require commitment solely to one discipline. 


\section{Groups}

According to Steven Johnson, author of "Where Good Ideas Come From,"7 an individual's hunch takes a long time to evolve into a useful and successful idea and this evolution is pushed along when it collides with the hunches of other people. People cannot always predict when this will happen—sometimes the best collisions surprisingly appear in a lecture hall, in a café or park, on the internet, through a book, or sitting on the front stoop of a home.

The likelihood of "good collisions" increases if people seek out opportunities to interact with others and work in groups and on teams with people who have different knowledge and experiences. If a university is to embrace transdisciplinary approaches, it will need to facilitate the formation and maintenance of interactions between people and ideas.

Teamwork is not easy, and requires individual and organizational effort to succeed. Scott Reeves, a professor at the University of California at San Francisco School of Nursing, studied health care clinical "teams" and found that few real teams actually exist. ${ }^{8} \mathrm{He}$ observed that often colleagues who are supposed to be on a team are actually just working alone, side by side. Reeves calls this "parallel play", drawing the analogy to children who play next to each other but are absorbed in their individual activities. Colleagues often believe they are engaging in excellent teamwork, but really their interactions are often fragmented and transient rather than synergistic and aligned around common goals.

There are growing efforts to methodically identify aspects and components of a good team and facilitate actions to make teams more successful. Team science, for example, is a relatively new field that has emerged to examine how teams organize, communicate, and produce work so that ultimately teams will be able to optimally manage these factors for better outcomes. ${ }^{9}$ If successful, the work of teams can have an overall collective impact that is larger than the sum of the impact of the individuals working within their individual disciplines.

A transdisciplinary university would put significant effort and resources into understanding and supporting teams, and students and faculty would face very little resistance when trying to form teams from different disciplines. Ultimately, universities would step beyond supporting isolated instances of teamwork and collaboration to developing structures that allow its students and faculty to make a larger collective impact. According to John Kania and Mark Kramer, supporting collective impact would mean supporting "a centralized infrastructure, a dedicated staff, and structured processes" that allow individuals and groups with a common agenda, shared measurement tools and indicators, regular communication, and mutually reinforcing activities to thrive in partnership." ${ }^{10}$ Of course, such an environment also needs to imbue the values of healthy argument and debate, respect for heterogeneous views, tolerance to a diversity of views and disciplines, and a mature acceptance of uncertainty. 


\section{How can people expand transdisciplinary universities?}

At the 2012 meeting, participants were challenged to think about how universities can create programs that include all the factors set forth above. Universities have a lot of potential for becoming transdisciplinary environments. Some of the strengths discussed at the 2012 meeting include:

- Co-location of many disciplines.

- $\quad$ Enthusiastic, curious, and influential students who are eager to try new things. Many are at the beginning of their careers and thus are not stuck in professionspecific language and concept ruts.

- $\quad$ Safe, protected environments for testing ideas and models. Work done in a university has limited negative impacts on people, economies, and societies.

- Inherent value of ideas. Unlike other organizations, projects tried in universities do not need to immediately yield a positive financial return on investment. The currency of the university is the idea, its articulation, its testing, and its application.

- Independence and credibility. Universities are seen as being relatively free of conflicts of interest, especially commercial interests.

- $\quad$ Respect for academics and the qualities it takes to achieve an academic position. (On the other hand, to some people, academics are thought of as abstract thinkers who do not know how to make things work in the real world.)

Of course, there are also limitations to how transdisciplinary a university can be. For example, universities are not a microcosm of the globe. They represent the most formally educated, relatively wealthy sample of the world's communities, and are often insular and disconnected from the local communities that surround them. Universities may also foster a reasonable amount of "groupthink" based on political or other shared perspectives, and sometimes can be slow to change. Finally, university strategy, structure, organization, processes, and rewards often can hinder transdisciplinary work. When working to change university environments to become more transdisciplinary, leaders should bear in mind these limitations and be modest about the extent to which changing a university will change a society.

\section{Barriers}

Though creating transdisciplinary environments sounds good conceptually, implementation can be very challenging. Many academics have had a hard time pursuing work that spans disciplines because the value of this work is not reflected in the supports, requirements, and incentives given to university faculty and students. The following are some of the barriers discussed at the 2012 meeting that academics have faced when trying to initiate or engage in transdisciplinary work.

- $\quad$ Too much division by categorical discipline. Disciplines are human-made categories that enable people to break down problems into more manageable 
parts to solve complex problems. They emerge from societal needs, labor markets, economics, population dynamics, understandings of efficiency and productiveness, and understandings of relationships. There are various types of disciplines that reflect the ways people tend to approach problems: by method (mathematically, philosophically), by topic or problem (public health, education, environmental studies), or by geography or culture (African studies, Russian literature). While disciplines are helpful, they may prevent individuals from seeing new relationships and connections, especially if one stops questioning his or her limits for the sake of group harmony.

- University priorities. Much of the success of university faculty or students pursuing academic careers lies in their ability to publish papers and get grants that bring money and recognition into the university. Currently, universities reward prolific publishing (particularly as lead or senior author) and funding, not necessarily collaboration and development of creative ideas. This value system may reward a powerful inner circle, but not those who are trying innovative ideas or activities on the margins.

- $\quad$ Funding silos. Many funding agencies, foundations, and professional societies tend to identify or be subdivided by subject or discipline (for example, the National Institutes of Diabetes and Digestive and Kidney Diseases; the National Eye Institute; the American Heart Association; the American Chemical Society). ${ }^{11}$ Many universities are now giving internal seed money to bring different groups together and the U.S. National Institutes of Health (NIH) is funding transdisciplinary team science. However, these opportunities are the exception. Furthermore, the type of research done by transdisciplinary teams may use unconventional methods (at least according to some disciplines) and adventurous post-hoc examination of data to make discoveries, and this does not fit easily into grant proposals that require more detailed preliminary hypotheses or preparation.

- $\quad$ Publishing constraints. Current publishing structures hinder transdisciplinary work in several ways. First, papers that span disciplines don't always have a clear publishing avenue since journals and reviewers often focus on specific disciplines. Second, authorship guidelines for papers assign the most value to first (lead) and last (senior) authors, and render the middle authors less known.

- Identity crisis and career tracks. Personal and professional identify and security is derived in part from having a clear sense of identity. ${ }^{12}$ People often seek to identify themselves with one discipline (sociologist, physicist, film producer etc.), which allows a clearer sense of self and a clearer path to a good future and success. Rewards and careers often tend to follow disciplines: professor of physics, Nobel Prize in Mathematics, etc. Academic tenure systems and career progress in industry tend to measure success in specialist terms. ${ }^{13}$ Additionally, faculty and students who don't fit neatly into disciplines have a much more difficult time finding a home in which to pursue their interests. It is more difficult 
for them to get promotions or tenure, or to be given as much recognition by leadership as their counterparts who specialize in more traditional ways.

- Institutional structural/logistical difficulties. Departments focus on specific disciplines, and it is challenging to share staff and resources across departments, or support financial and administrative processes between departments. Permitting these structural outliers can also go against the institution's strategy and pull existing organizational design aspects out of alignment. ${ }^{14}$ In addition, it is difficult for specialists to find time to do transdisciplinary work while still finding time to learn their disciplines. Lastly, students and fellows leave every few years, making it difficult to sustain new student-driven transdisciplinary programs.

- Difficult teamwork logistics. Stepping out of the comfort zone of a discipline takes effort-teams need to translate field specific languages and integrate different types of thinking into a coherent solution. Often teamwork takes more time and effort and can be more costly than working alone. Furthermore, merely forming a team can be difficult if parts of problems that will entice multiple disciplines to contribute have not been identified.

- $\quad$ Lack of measures. History and experience has shown that there is value in transdisciplinary work. But, how does one prove this to administrators or funders? The problems that transdisciplinary thinkers and teams excel at are complex in the first place, and showing the impact of the team itself on the problem-solving process is yet another complexity. As mentioned previously, current measures of success tend to focus on publication authorship and grants, not productive teamwork. Coming up with clear measures of teamwork success is critically important to show value to the administrative power structure.

- Lack of mentors. Mentors tend to associate with their own specific disciplines or subject areas, and often encourage mentees to follow the safe path of sticking to single disciplines or to 'keep focus'. They may resist what they perceive as compromises to the rigors of their discipline to accommodate transdisciplinary work. Even when supportive of the idea, mentors may also find it challenging to help a mentee bridge disciplines, or to be a co-mentor with another individual from another discipline. Transdisciplinary science needs permeable borders across disciplines, which may require less structured mentorship, with mentors who appreciate other disciplines and can encourage and support their mentees to discover the excitement that may reside in the connections between disciplines. ${ }^{15}$

\section{Moving Forward}

The barriers are large, but not insurmountable. In many ways, overcoming these barriers to transdisciplinary work in of itself needs active transdisciplinary thinking. Administrators, students, and faculty should move beyond just getting people in different disciplines to work together, and should continue to push on for structures and support that facilitate long-term and continuous transdisciplinary work. 
During the 2012 meeting and afterwards, participants spent a large amount of time brainstorming activities and processes that would push universities toward becoming more transdisciplinary. Many of the ideas in Figure 1 reflect activities that universities are already developing and implementing, and others are activities that universities and other actors should consider adopting. Many of these activities are especially relevant to global health education, and will be vital in the training of students who aspire to tackle complex health problems.

In his essay The Loss of the University, farmer, writer, and former professor Wendell Berry discusses the university's role in assisting the development of human beings who are "responsible heirs and members" of their communities and culture. He writes, "Underlying the idea of a university - the bringing together, the combining into one, of all the disciplines -is the idea that good work and good citizenship are the inevitable by-products of the making of a good — that is, a fully developed —human being. This, as I understand it, is the definition of the name university." 16 As the problems faced in communities and the world become ever more complex and influenced by more and more interrelated factors, universities will need to ground learners in this unifying principle, not divide people into ever more separate parts, with separate methods and separate interests and separate achievements. The discussed proposals for institutional changes have the potential to, whether in small ways at first or in bold steps, lead to more space and support - even a shift in mentality - to transform how the university contributes to the growth and wellbeing of individuals and communities around the world.

\section{Endnotes}

1. Jefferson, T. Jefferson to Hugh L White and Trustees of the Lottery for East Tennessee College, May 6, 1810. In: Jefferson Looney, J., editor. The Papers of Thomas Jefferson: Retirement. Princeton: Princeton University Press; 2005. p. 365Series vol. 2

2. Participants were purposefully selected to represent an array of disciplines, stages of training, and institutions. They were people who had taken an active interest in making their universities more transdisciplinary through projects and programs, new collaborations, and administrative and curricular changes. The meeting participants were: Mohammed Ali (Emory), Rebecca Baggett (Emory), Satchit Balsari (Weill Cornell, Harvard), Asaf Bitton (Brigham and Women's, Harvard), Nisha Botchwey (Georgia Tech), Leigh Carroll (IOM), Patricia Cuff (IOM), Dan Eisenberg (University of Washington), Dave Frisvold (Emory), Hank Gardner (Colorado State), Roger Glass (Fogarty International Center), Matthew Goodwin (Weill Cornell), Mark Huffman (Northwestern), Amanda Jones (Emory, CDC), Bridget Kelly (IOM), Sandeep Kishore (Weill Cornell), Jeff Koplan (Emory), Gretchen Martin (Emory), Suzanne Mason (Emory), William Masters (Tufts), Martha McGill (Yale), Molly McMahon (Mayo Clinic), Rob Murphy (Northwestern), K.M. Venkat Narayan (Emory), Rachel Nugent (University of Washington), Karen Siegel (Emory), Maryam Shafee (Weill Cornell), Lisa Shufro (TEDMED), Rachel Taylor (IOM), Rajesh Vedanthan (Mount Sinai), Roseanne Waters (Emory), Collin Weinberger (IOM, Johns Hopkins), Heather Wipfli (University of Southern California), Samantha Woo (Northwestern), Cathy Yeckel (Yale)

3. Rosenfield PL. The potential of transdisciplinary research for sustaining and extending linkages between the health and social sciences. Social Sciences and Medicine. 1992; 35(11):134357.Institute of Medicine. Establishing Transdisciplinary Professionalism for Improving Health Outcomes: Workshop Summary. Washington, DC: The National Academies Press; 2014.

4. B. Russell (attributed: source unknown)

5. A. Einstein (attributed: source unknown). 
6. Taimina, D. Crocheting Hyperbolic Planes. presented at TedxRiga 2012; Riga, Latvia. June 14, 2012; available at <http://tedxtalks.ted.com/video/Crocheting-hyperbolic-planes-Da;search\%3Atag $\% 3 \mathrm{~A} \% 22$ latvia\%22>

7. Johnson, S. Where Good Ideas Come From. presented at TEDGlobal 2010; Oxford, UK. July 13, 2010; available at <www.ted.com/talks/steven_johnson_where_good_ideas_come_from>

8. Institute of Medicine. Assessing Health Professional Education: Workshop Summary. Washington, DC: The National Academies Press; 2014.

9. Northwestern University, Northwestern University Clinical and Translational Sciences Institute. COALESCE: CTSA Online Assistance for Leveraging the Science of Collaborative Effort. at <http://teamscience.net/> (last visited June 6, 2014)National Cancer Institute. Team Science Toolkit. at < https://www.teamsciencetoolkit.cancer.gov/Public/Home.aspx> (last visited June 6, 2014)SciTS. Building the knowledge base for effective team science. at <http:// www.scienceofteamscience.org/> (last visited June 6, 2014)

10. Kania J, Kramer M. Collective Impact. Stanford Social Innovation Review. Winter;2011 9(1) available at <www.ssireview.org/articles/entry/collective_impact>.

11. Narayan, KMV. Ten Barriers to Trans-disciplinary Science. BMJ Group Blogs. at <blogs.bmj.com/bmj/2012/08/06/k-m-venkat-narayan-ten-barriers-to-trans-disciplinary-science/> (last visited May 1, 2014)

12. Id.

13. Id.

14. Galbraith, JR. Designing Organizations: An Executive Guide to Strategy, Structure, and Process. San Francisco: Jossey-Bass; 2002.

15. See Narayan, supra note 11 .

16. Berry, W. Home Economics. New York: North Point Press; 1987. The Loss of the University; p. 77-97.p. 77

17. See Institute of Medicine, supra note 4.

18. Fogarty International Center. Framework Programs for Global Health Innovation. at <www.fic.nih.gov/programs/pages/framework-innovations.aspx> (last visited May 1, 2014)

19. Emory Global Health Institute, Emory University. Emory Global Health Case Competitions. available at <globalhealth.web.emory.edu/what/student_programs/case_competitions/index.html> (last visited May 1, 2014)

20. The University of Oklahoma-Tulsa, School of Community Medicine. Summer Institute Field Book. 2013. available at < https://www.ou.edu/content/tulsa/community_medicine/2013/ curriculum---schedule.html> (last visited May 14, 2014)

21. School of Public Health, Yale. Slide Show: The Art of Public Health. at <publichealth.yale.edu/ news/slideshows/posters.aspx> (last visited May 1, 2014)

\section{Biographies}

Leigh Carroll is a Research Associate at the Institute of Medicine, where she works on chronic disease prevention projects and provides research support to the IOM president.

Mohammed K. Ali, MBChB, MSc, MBA, is faculty in the Departments of Global Health and Epidemiology at Emory University and an advisor for the Centers for Disease Control's Division of Diabetes Translation.

Patricia Cuff is the Director of the Institute of Medicine's Global Forum on Innovation in Health Professional Education. 
Mark D. Huffman, MD, MPH, is an Assistant Professor of Preventive Medicine and Medicine-Cardiology at Northwestern University and a practicing cardiologist with an interest in global cardiovascular epidemiology, prevention, and outcomes research.

Bridget Kelly, MD, $\mathrm{PhD}$ is a Senior Program Officer at the Institute of Medicine, working on projects that cover a wide range of health and education topics.

Sandeep P. Kishore, MD, PhD is Yale internal medicine resident and leader of the Young Professionals Chronic Disease Network (YP-CDN).

K. M. Venkat Narayan, MD, MSc, MBA is a physician-scientist, the Director of Emory Global Diabetes Research Center, and is the Ruth and O.C. Hubert Chair of Global Health, and professor of epidemiology \& medicine at Emory University.

Karen R. Siegel, MPH, is a PhD student in Nutrition and Health Sciences and a Woodruff Scholar and ARCS Scholar at Emory University's Laney Graduate School and Rollins School of Public Health.

Rajesh Vedanthan, MD, MPH, is and Assistant Professor of Medicine (Cardiology) and Health Evidence and Policy at the Zena and Michael A. Wiener Cardiovascular Institute, Mount Sinai Medical Center, New York, NY 

Reflect the value of transdisciplinary work in rewards and measurements.
- Develop incentives and rewards for leaders and projects.
o Obtain commitments from major funders and presidents of universities.
- Elect Institute of Medicine (IOM)/National Academies of Science/professional society members in recognition of their transdisciplinary work rather than specialization.
- Create a Nobel Prize-like global award that is specifically for innovations created by transdisciplinary teams
o Develop ways of measuring the value of transdisciplinary work.
o Evaluate transdisciplinary projects, teamwork, and approaches.

\section{Make team work easier.}

Logistics:

$\circ$ Develop a social contract/code of ethics that binds disciplines and is trusted by the public (e.g. IOM's Global Forum on Innovation in Health Professional Education is working on anchoring health professionals around a unified code of ethics ${ }^{17}$ ).

o Provide discipline-neutral locations for collaboration.

- Be conscious of discipline-specific jargon.

$\circ$ Provide incentives (e.g. additional time, resources) for choosing to work with a team. Publications:

$\circ$ Designate journal issues for transdisciplinary work or create new, transdisciplinary journal.

o Address authorship concerns (e.g. for some teams, first/last author guidelines do not make sense).

Funding:

o Hold meetings for potential funders to share ideas on supporting transdisciplinary work. o Track available funding for transdisciplinary work.

- Develop transdisciplinary requirements for research grants. (E.g. Fogarty International Center grants have required applicant teams to represent multiple disciplines. ${ }^{18}$ )

o Provide funding that is outcome-based and flexible.

o Require that transdisciplinarity be considered in project and program planning, implementation, and evaluation as a condition of research funds.

Allow for shifts in attitude

$\circ$ Discourage intimidation and dismissiveness; and encourage people to follow their hunches to expose new connections.

- Develop a culture where students and faculty are not afraid to admit uncertainty. 


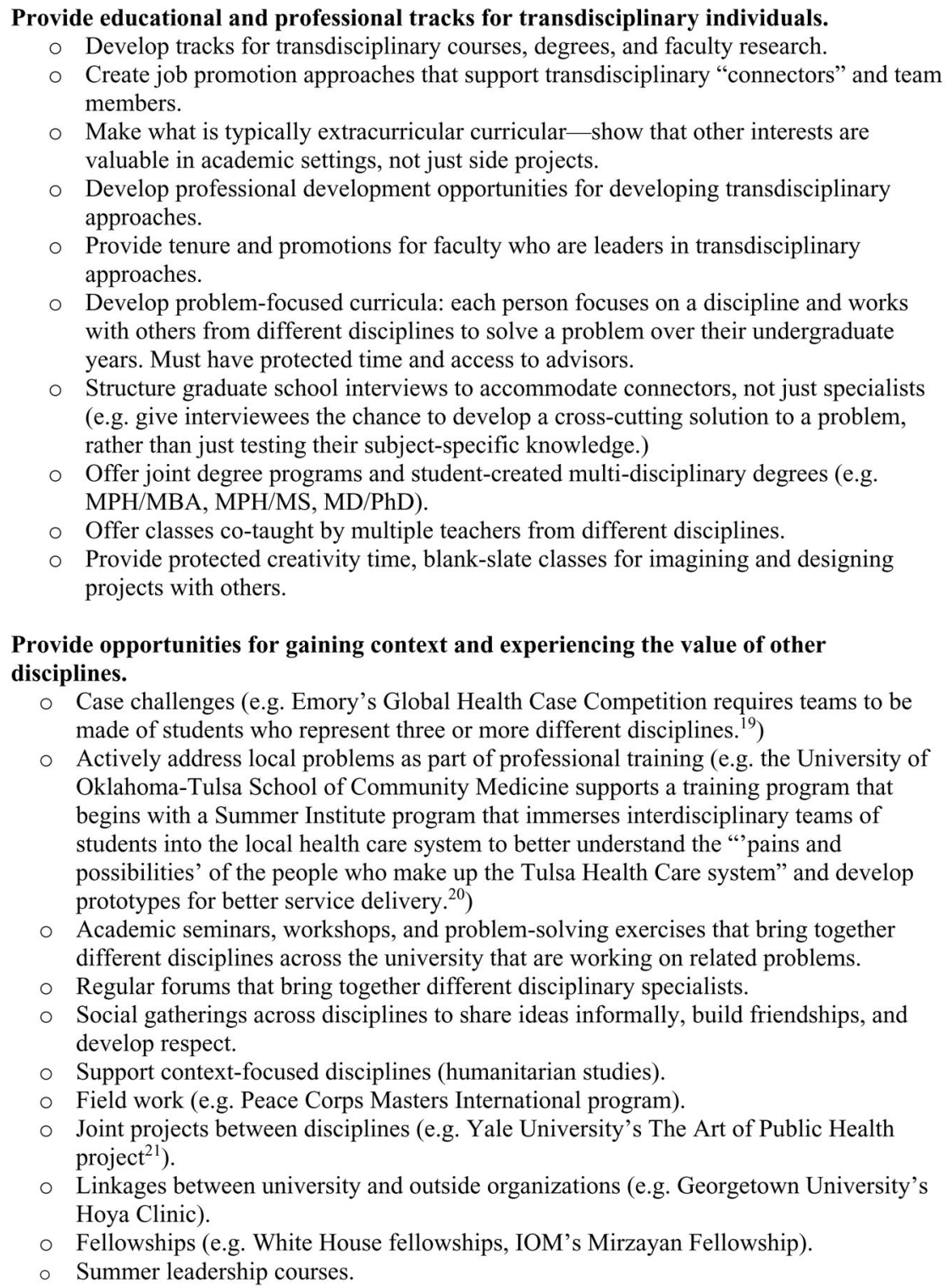

- Make what is typically extracurricular curricular - show that other interests are valuable in academic settings, not just side projects.

- Develop professional development opportunities for developing transdisciplinary approaches.

- Provide tenure and promotions for faculty who are leaders in transdisciplinary approaches.

- Develop problem-focused curricula: each person focuses on a discipline and works with others from different disciplines to solve a problem over their undergraduate years. Must have protected time and access to advisors.

- Structure graduate school interviews to accommodate connectors, not just specialists (e.g. give interviewees the chance to develop a cross-cutting solution to a problem, rather than just testing their subject-specific knowledge.)

- Offer joint degree programs and student-created multi-disciplinary degrees (e.g. $\mathrm{MPH} / \mathrm{MBA}, \mathrm{MPH} / \mathrm{MS}, \mathrm{MD} / \mathrm{PhD}$ ).

- Offer classes co-taught by multiple teachers from different disciplines.

- Provide protected creativity time, blank-slate classes for imagining and designing projects with others.

Provide opportunities for gaining context and experiencing the value of other disciplines.

○ Case challenges (e.g. Emory's Global Health Case Competition requires teams to be made of students who represent three or more different disciplines. ${ }^{19}$ )

- Actively address local problems as part of professional training (e.g. the University of Oklahoma-Tulsa School of Community Medicine supports a training program that begins with a Summer Institute program that immerses interdisciplinary teams of students into the local health care system to better understand the "pains and possibilities' of the people who make up the Tulsa Health Care system" and develop prototypes for better service delivery. ${ }^{20}$ )

- Academic seminars, workshops, and problem-solving exercises that bring together different disciplines across the university that are working on related problems.

- Regular forums that bring together different disciplinary specialists.

- Social gatherings across disciplines to share ideas informally, build friendships, and develop respect.

- Support context-focused disciplines (humanitarian studies).

- Field work (e.g. Peace Corps Masters International program).

- Joint projects between disciplines (e.g. Yale University's The Art of Public Health project $^{21}$ ).

- Linkages between university and outside organizations (e.g. Georgetown University's Hoya Clinic).

- Fellowships (e.g. White House fellowships, IOM’s Mirzayan Fellowship).

- Summer leadership courses.

Figure 1.

Transdisciplinary Activities and Processes 